\title{
Patterns of Antemortem Tooth Loss in Late Prehistoric West- central Tennessee
}

\author{
Maria Ostendorf Smith ${ }^{1 *}$ and Tracy K. Betsinger ${ }^{2}$ \\ ${ }^{1}$ Illinois State University \\ 2 State University of New York, Oneonta
}

Keywords: Mississippian, maize, Vacant Quarter, Tennessee

\begin{abstract}
The later prehistoric subsistence-settlement pattern in the Kentucky Lake Reservoir (KLR) of northern west-central Tennessee is of interest as human occupation inexplicably terminates by AD 1450 as part of a larger regional depopulation. Antemortem tooth loss (AMTL) collectively and by tooth type was identified in four site samples from the KLR. These are a Late Woodland (AD 600-900) sample (Hobbs) and three Middle Mississippian period (AD 1100-1400) hierarchically organized and presumptively maize agriculturalist samples (Link/Slayden, Gray Farm, Thompson Village). AMTL prevalence in the Hobbs sample is consistent with a native crop and seasonal foraging economy. The AMTL in the Link/Slayden sample is more congruent with the pre-maize Late Woodland sample than the essentially contemporaneous Gray Farm site sample. Thompson Village, a later-dated satellite community of the Gray Farm polity, exhibits significantly fewer AMTL than Gray Farm. This may flag climate-influenced agricultural shortfall of dietary carbohydrates later in the occupation sequence. Additionally, males in the Gray Farm site sample have significantly more AMTL than males in the other two Mississippian samples. The patterns suggest regional, possibly shortfall mitigated, differences in maize intensification with a polity-specific male-focused maize consumption in the Gray Site.
\end{abstract}

Oral pathology has frequently been an effective barometer of community health and an attestable marker of subsistence strategy in archaeological contexts where material culture provides the interpretive context (e.g., Betsinger \& Smith, 2018;

Larsen, 1983; Larsen et al., 1991; Lukacs, 1992; Russell et al., 2013; Turner, 1979; Watson, 2008). Maize is a cariogenic carbohydrate (e.g., Bibby et al., 1951; Horton et al., 1985; Newbrun, 1979). Caries has been a particularly interpretively useful oral pathological condition in North America, enabling the flagging of maize-intensive agriculture (e.g., Emerson et al., 2005; Larsen, 1981; Powell, 1985; Watson, 2008). In the absence of adequate oral hygiene, crown or cervical carious lesions progress to penetrate the pulp cavity resulting in dental necrosis and ultimately to exfoliation of the tooth from the alveolar anchor. In more extensive assessments of caries prevalence (i.e., caries correction factor), antemortem tooth loss (AMTL) has been included as a proxy for carious teeth (Duyar \& Erdal, 2003; Erdal \& Duyar, 1999; Lukacs, 1995; MarquezGrant, 2009).

Although AMTL is primarily attributed to the destructive pathogenesis of caries and periodontal disease (Baelum et al., 1986; Kida et al., 2006; Müller \& Hussein, 2017; Niessen \& Weyant, 1989; Ong, 1998; Shaffer et al., 2013; van der Velden et al., 2015), there are other causes. These include rapid attrition, ablation, acidogenic response, and traumatic injury (Costa, 1980; Duyar \& Erdal, 2003; Han \& Nakahashi, 1996; Humphrey \& Bocaege, 2008; Lukacs, 2007; Nelson et al., 1999; Niessen \& Weyant, 1989; Pollard et al., 1997). Although traumatic injury may be a contributing factor in a few cases of AMTL in the late prehistoric samples from west-central Tennessee, the present study observed that attrition and ablation, as elsewhere in the Tennessee Valley (Smith, 1982), are absolutely not evident. As such, oral pathology is the most apparent contributor to AMTL. Previous assessment of caries prevalence in Late Prehistoric human osteologi-

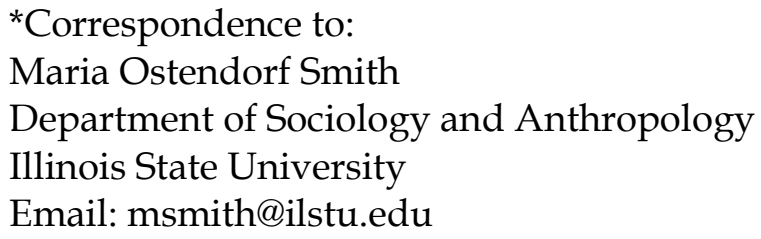


cal samples from northern west-central Tennessee did not identify a clear temporal trend of caries increase with agriculturalization suggesting regional variability in the reliance of maize relative to domesticated native seed crops (Smith \& Betsinger, 2019), a not uncommon phenomenon elsewhere in the Mississippian world (e.g., Hart \& Lovis 2013; Hutchinson et al., 1998; Scarry, 1993). A renewed look at oral health using AMTL data and a larger sample of sites may help clarify the lack of comparative oral health congruence with maizeintensive samples. Given the inexplicable abandonment of the region by AD 1450 as part of the large scale depopulation of the lower Ohio River Valley ("The Vacant Quarter") (Cobb \& Butler, 2002; Williams, 1990), the temporal and regional differences in AMTL may also provide some eco-political insight.

The maize-intensive subsistence economy in the eastern United States temporally associates with the Mississippian period ( AD $1000-1500)$ and life way, the apex of which (AD 1200-1400) is sociopolitically characterized as having centralized (possibly chiefdom level) authority, a complex iconographic-rich cosmology, a well-organized aggregated village settlement pattern, shelltempered pottery, and wall trench architecture (Bense, 2016; Cobb, 2003; King, 2002; King et al., 2007; Lewis et al., 1998; Peregrine 2013; Wilson, 2017). Since the Mississippian period material culture correlates are present in the late prehistory of upper west-central Tennessee, a maize-intensive agricultural subsistence economy has been proposed (Bass, 1985; Dye, 2004, 2007; Krus \& Cobb, 2018; Lunn, 2013; Mainfort, 1996). The purpose of this assessment is to clarify why the Mississippian period Kentucky Lake collective site sample from Link [40HS6] and Slayden [40HS1] did not exhibit a caries frequency or pattern consistent with unequivocal maize-intensive comparative samples from Tennessee (Smith \& Betsinger, 2019).

\section{Materials and Methods}

Archaeological context

In eastern North America, the time period spanning the Late Woodland ( AD 600-900) to the apex of the subsequent Mississippian period ( AD12001400 ) is one of marked regionally and temporally variable economic, settlement, and sociopolitical change (Bense, 2016; VanDerwarker et al., 2017; Fritz, 1990; Nassaney, 2001; Scarry, 1993; Wilson \& Sullivan, 2017). The subsistence-settlement pattern of the Late Woodland is generally characterized by dispersed small villages within circumscribed terri- tories that economically engaged in cultivating native seed crops as well as foraging strategies (i.e., the Eastern Agricultural Complex) (Bense ,2016; Fritz, 1990; Nassaney, 2001; Scarry, 1993). This pattern is archaeologically evident in central Tennessee (Shea, 1977). The geographic distribution and the ensuing time period of the Mississippian is defined by the transition to maize-reliant agriculture (Bense 2016; Fritz, 1992; Krus \& Cobb, 2018; Lewis et al., 1998; VanDerwarker et al., 2017), palisaded aggregated villages with one or more variably functioning (e.g., domiciliary, mortuary, temple) platform mounds flanking a central plaza, and more complex, likely hierarchical, social organization (Bense, 2016; King \& Freer, 1995; Schroedl, 1998). In the greater Southeast, the Mississippian period is also iconographically rich (i.e., "Southeastern Ceremonial Complex" or "Southeastern Ceremonial Exchange Network") (Bense, 2016; King et al., 2007).

The typical Mississippian large aggregated settlement was organized around a central plaza that was flanked by flat-topped mounds which variably served as platforms for domiciles, temples, mortuary structures (e.g., charnel houses, mound burials), or community buildings (Bense, 2016; Lewis et al., 1998; Nash, 1968). Mound settlements were civic-ceremonial polity centers geographically surrounded by satellite communities and separated from other mound-centered polities by buffer zones (Bense, 2016; Dye ,2004; Hally, 1993).

The sites of Gray Farm (40SW1), Thompson Village (40HY5), Link Farm (40HS6)/Slayden (40HS1), and Hobbs (40HS44) are located in the Lower Tennessee River Valley of west-central Tennessee in the projected catchment area of what is now the Kentucky Lake Reservoir (KLR) (Figure 1). The sites were excavated between 1938 and 1944 as part of extensive salvage archaeological recovery operations conducted by TVA (Tennessee Valley Authority) and the Federal WPA (Works Progress Administration) program prior to the completion of the Kentucky Dam (Gilbertsville, Kentucky) (Dye, 2013). This section of the Tennessee River valley lies between the Western Tennessee Uplands on the west, and the steep hills of the Western Highland Rim on the east. The archaeological recovery of the sites' material culture and skeletal material was hampered by time constraints, many years of agricultural activity, highway construction, and extensive looting (Bass, 1985). There are no monographic site reports for any of the five sites, but one or other of them has been the source of descriptive or synthetic archaeological assess- 
ment (Bass, 1985; Dye, 2002; Lunn, 2013). Given the absence of carbon samples, the sites are relatively dated (ceramic sequences, mortuary patterning) (Bass, 1985).

The five site samples examined here consist of the Late Woodland period ( AD 500-900) site of Hobbs (Kuemin Drews, 2001) and four primarily Middle Mississippian period ( AD 1100-1350) sites that segregate into two geographically discrete polities (Bass, 1985; Dye, 2004). Hobbs is a mound mortuary site on the main channel of the Tennessee River downstream from the Link and Slayden sites (Figure 1). The Early-to-Middle Mississippian period sites of Slayden ( AD 1050-1250) and Link Farm ( AD 1250-1400) are located on opposite shores of the Duck River floodplain, geographically close to the confluence with the Tennessee River (see Figure 1) (Dye, 2002, 2007; Kuemin Drews, 2000; Lunn, 2013). The multiple mound site of Link Farm is argued to have expanded from the Slayden village site with the former becoming the administrative center for the Middle Mississippian period Link Farm polity (Dye, 2002, 2004; Lunn, 2013;

Nash, 1968). Given the paucity of human remains recovered from Slayden, the site samples are combined for this study as Link/Slayden.

The Gray Farm polity is located near the confluence of the Big Sandy River and the Tennessee River (see Figure 1). The sites included here are the multiple mound Gray Farm site ( AD 1150-1400) (Dye, 2004) and the Thompson Village site which fissioned from the former during the late Middle Mississippian period ( post AD 1250) and remained occupied through the early Late Mississippian period (Bass, 1985) ( AD1350-1450). All aboriginal occupation of west-central Tennessee apparently ended before circa AD 1450 (Bass, 1985; Smith, 2010) as part of the phenomenon of Middle Mississippian period regional abandonment in the greater lower Ohio River Valley (the "Vacant Quarter") (Cobb \& Butler, 2002; Krus \& Cobb, 2018; Williams, 1983). The specific reasons for the abandonment are unclear, but interpretations include corollaries of climate change (i.e., the "Little Ice Age" [ AD1400-1700]) (Grove, 2012; Mann et al., 2009, Meeks \& Anderson, 2013) such as resource depletion, and/or endemic warfare (Bass 1985; Krus, 2013; Worne, 2011).

Although the Kentucky Lake Mississippian period site samples archaeologically exhibit the hallmarks of Mississippian settlement organization and iconography, they are not further sub-divisible into temporally and regionally defined cultural phases. This is partly an artifact of pre-excavation site disturbance which impeded stratigraphic control. Geographically they are west of the arguably aboriginal interaction-impeding Western Highland Rim geophysical feature as well as outside of the

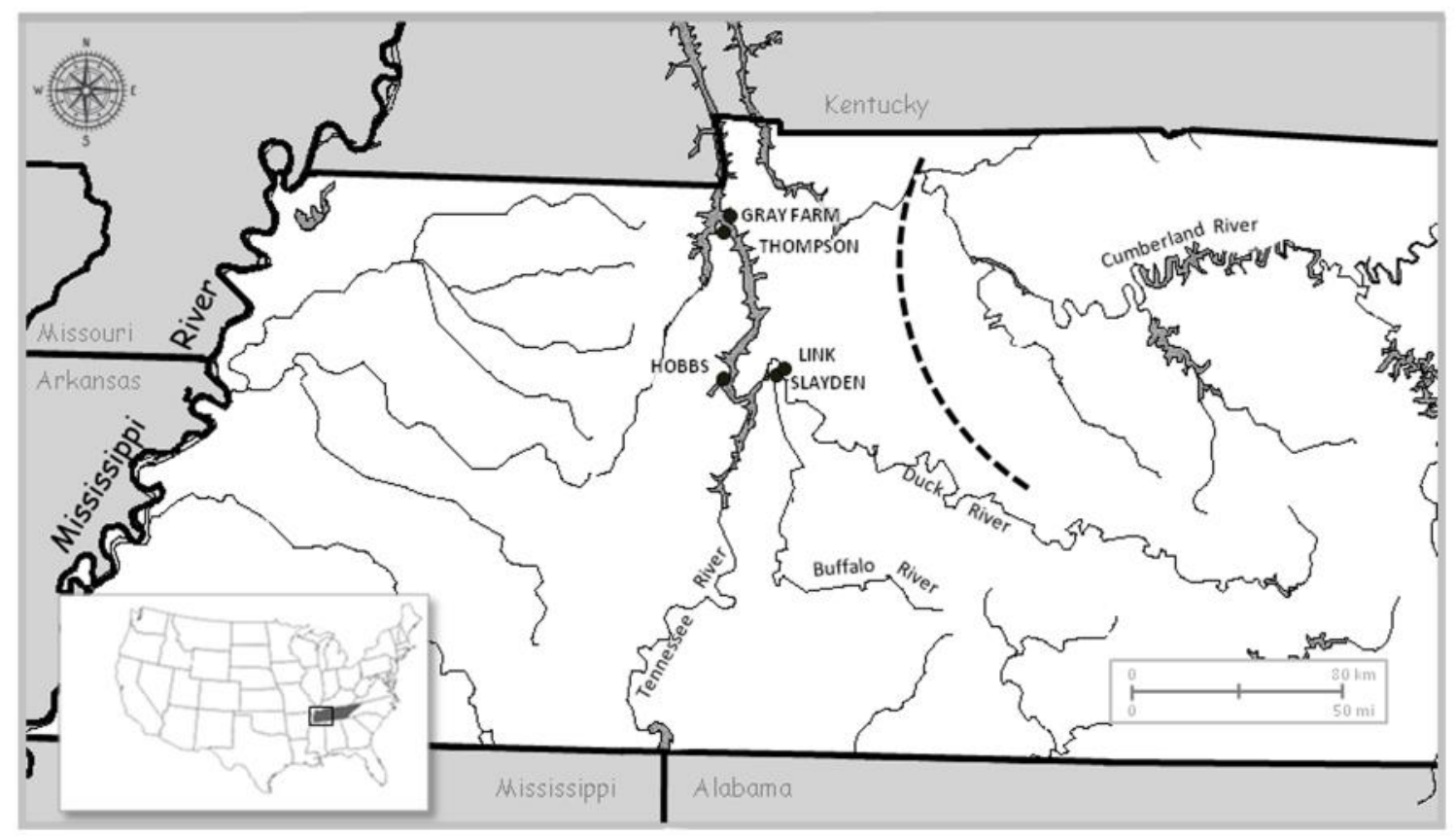

Figure 1. Map of the Kentucky Lake Reservoir sites utilized in the study. The western boundary of the Mississippian Period (AD 1000-1450) Middle Cumberland Culture is indicated by the dashed line. 
Mississippian period ( AD 1050-1450) Middle Cumberland Region (MCR) (see Figure 1) of the central Cumberland River valley and its tributaries (Beahm, 2013; Dowd, 2008; Moore \& Smith, 2001; Smith, 1992). There is also no archaeological evidence that the KLR samples had contact with Mississippian period sites in the Mississippi River drainage of west Tennessee (Mainfort, 1996; Mainfort \& Moore, 1998). However, some shared cultural elements with the MCR (Dowd, 2008; Ferguson, 1972; Smith, 1992) are evident (e.g., infants interred within house structures and at least some use of limestone slabs to line graves (i.e., stone box burials) (Bass, 1985; Wamsley, 2018).

\section{Sample and Diagnostic criteria}

The skeletal age at death and differentiation by sex were previously determined using standard nonmetric osteological protocols (Buikstra and Ubelaker, 1994) and the information is archived in a computer data base. The individuals regarded as adults in the AMTL assessment had least two of the third molars in occlusion in vivo. Mandibles and maxillae were the primary source of AMTL, but loose teeth were included if antimeres were present or if they were accounted for by alveolar bone (either as sockets or remodeled alveolar processes). Individ- uals assessed consisted of the numbered burials; unaffiliated or extraneous teeth were excluded. Adults were segregated into three age-at-death categories: young ( $\sim 18$ years to $\sim 35$ years), middle age ( $\sim 35$ years to $\sim 50$ years of age), and old age $(50+)$ years of age (Table 1$)$.

Teeth were considered present when observed in situ, as loose teeth, and as un-remodeled alveolar sockets. Teeth were considered lost antemortem if the socket was porotic and failed to conform to the sharp alveolar contours of an in situ root, displayed abscessing with no accompanying (loose) tooth, and ultimately, by the remodeling/ resorption of the alveolar corpus. Teeth were consolidated by arch and antimere and evaluated for AMTL by categories of tooth type. Incisors and canines were collapsed into a single category (I/C), as were all premolars (PM). Molars were assessed by number (M1, M2, M3) and collectively.

Preservation ranged from fair to poor for all sites limiting the primary assessment to postmortem presence by tooth type rather than pattern(s) of AMTL by individual (see Table 1). The Gray Farm sample is particularly poorly preserved; seventy percent of the interments with dental data are not ascribable to an age-at-death category and 23 individuals ( $\sim 38 \%)$ are assignable to a sex.

Table 1. Demographic overview of sample preservation and segregation by age-at-death and biological sex.

\begin{tabular}{|c|c|c|c|c|c|c|c|c|}
\hline \multicolumn{9}{|c|}{ LINK FARM/SLAYDEN (Total n = 25²) } \\
\hline \multicolumn{3}{|c|}{ young } & \multicolumn{3}{|c|}{ m aged } & \multicolumn{3}{|c|}{ old age } \\
\hline males & females & sex-indet. ${ }^{1}$ & males & females & sex-indet. ${ }^{1}$ & males & females & sex-indet. ${ }^{1}$ \\
\hline 1 & 3 & 2 & 4 & 3 & 1 & 0 & 3 & 0 \\
\hline \multicolumn{9}{|c|}{ GRAY FARM $\left(\right.$ Total $\left.\mathrm{n}=61^{2,3}\right)$} \\
\hline \multicolumn{3}{|c|}{ young } & \multicolumn{3}{|c|}{ m aged } & \multicolumn{3}{|c|}{ old age } \\
\hline males & females & sex-indet. ${ }^{1}$ & males & females & sex-indet. ${ }^{1}$ & males & females & sex-indet. ${ }^{1}$ \\
\hline 0 & 1 & 0 & 9 & 2 & 2 & 2 & 2 & 0 \\
\hline \multicolumn{9}{|c|}{ THOMPSON $\left(\right.$ Total $\left.\mathrm{n}=103^{2,4}\right)$} \\
\hline \multicolumn{3}{|c|}{ young } & \multicolumn{3}{|c|}{ m aged } & \multicolumn{3}{|c|}{ old age } \\
\hline males & females & sex-indet. ${ }^{1}$ & males & females & sex-indet. ${ }^{1}$ & males & females & sex-indet. ${ }^{1}$ \\
\hline 8 & 14 & 1 & 16 & 19 & 3 & 5 & 8 & 0 \\
\hline \multicolumn{9}{|c|}{ HOBBS $\left(\right.$ Total $\left.n=8^{2}\right)$} \\
\hline \multicolumn{3}{|c|}{ young } & \multicolumn{3}{|c|}{$\mathrm{m}$ aged } & \multicolumn{3}{|c|}{ old age } \\
\hline males & females & sex-indet. ${ }^{1}$ & males & females & sex-indet. ${ }^{1}$ & males & females & sex-indet. ${ }^{1}$ \\
\hline 1 & 0 & 0 & 1 & 3 & 0 & 0 & 1 & 0 \\
\hline
\end{tabular}

${ }^{1}$ sex-indeterminate

${ }^{2}$ sample Total includes individuals not assignable by either age or sex

3 43/61 not ageable, 7/43 sex assignable but not ageable

${ }_{4}^{4} 29 / 103$ not ageable, 20/29 sex assignable but not ageable 
Therefore, the primary intersite assessments are based on the presence of teeth (loose and in situ), alveolar evidence (pre- and postmortem loss), and AMTL.

\section{Analytical methods}

Fisher's exact test (https:/ / www.graphpad.com) was used to test the prevalence differences for all teeth and by tooth category, and in the same categories when segregated by sex and/or age-atdeath. No tests were undertaken for samples of less than ten. Small sample sizes prevented several comparisons. For those tests meeting the minimum sample size, the results are considered tentative.

\section{Results}

The Late Woodland Hobbs mortuary sample has the lowest total tooth sample prevalence of AMTL $(14 / 153,9.1 \%)$ (Table 2$)$, but it is not significantly less $(p=0.4479)$ than the Middle Mississippian Link/Slayden sample (46/391, 11.8\% ) (Table 3). However, the Hobbs sample has significantly fewer teeth lost antemortem than the Middle Mississippian site samples of Gray Farm $(p=0.0001)$ and Thompson Village $(p=0.0160)$. The total tooth sample AMTL between the Gray Farm (32.7 and Thompson Village (16.7\%) site samples is also significantly different $(p=0.0001)$. When AMTL is further evaluated by the five tooth type categories, the Hobbs and Link/Slayden samples remain congruent (see Table 3). Hobbs differs in almost all tooth categories from Gray Farm and from the Thompson site sample in the collective loss of molars. Link/Slayden lost significantly fewer teeth than Gray Farm in all tooth categories except the first molar, and in many tooth categories compared to Thompson Village. Thompson, with overall fewer AMTL than the Gray Farm, differs from it in fewer incisiform and premolar tooth loss (see Table 3).

\section{Age-at-death comparisons}

Despite the case deficit in the young age at death (see Table 2), it is apparent that overall, the young adult cohorts for all four west-central Tennessee sites experience the lowest AMTL prevalence in each of the respective site samples. Where statistical tests are possible, there is a pattern of no intersite differences except for the greater AMTL of all molars in Gray Farm relative to Thompson (see Table 2). In the Middle Age cohort, the vulnerability of complex-crowned teeth to factors contributing to AMTL is evident. Hobbs and Link/ Slayden exhibit a significant difference in the loss of M1, the molar longest in occlusion, as well as molars overall and the dentition overall. Hobbs, however, has significantly fewer teeth lost in more categories than both Gray Farm and Thompson. The Link/Slayden site sample, in turn, has lost significantly fewer teeth than Gray Farm and molars compared to Thompson.

Thompson Village, with an overall tooth loss in the Middle Age category of $25 \% \quad(n=179 / 715)$, is significantly lower than Gray Farm (89/165, 54\%) in the loss of incisiform teeth (see Table 1) $(7.8 \%$ versus $36.5 \%, 13.8 \%$ versus $49 \%$ ), but congruent in the loss of individual molars. Meaning, the samples are evidently significantly different in the absolute number of molars lost, but not different in the proportion by molar type (see Tables 2 and 3 ). Few meaningful statistical comparisons were possible for many of the tooth categories in the Old Age cohort (i.e., samples < 10). All samples yielded few individuals in that age category (see Table 1) resulting in little available dental data.

Intrasite and intersite differences by sex

Site sample dissimilarities (see Tables 2 and 3) may be affected by sex-based differences in vulnerability to oral pathology (e.g., Lukacs \& Largaespada, 2006). The samples were first compared for overall AMTL differences by sex (Table 4). In the collective adult sample, there are no sex differences in AMTL in the Hobbs and Link/Slayden samples. There are multiple categories of sex difference in the Gray Farm and Thompson samples. In the Gray Farm sample, males exhibit significantly more AMTL $(p=0.0001)$ than females $(43.5 \%$ versus $20.8 \%)$ and more AMTL in the anterior teeth; in the Thompson Village sample, females have more AMTL than males ( $\sim 18 \%$ versus $12.6 \%)$ ( $p=0.0022)$. However, more tooth categories in the Thompson Village sample display the higher female AMTL prevalence.

Very few tooth class categories have a large enough Young Adult case sample to argue a pattern. However, the Gray Farm sample has more male AMTL for all molars and all dentition overall. Since most Young Adult site samples exhibit very few cases of AMTL (see Table 2) sex differences may be statistically undetectable. Sex differences do emerge in the Middle Age category (see Tables 4 and 5). At the Link/Slayden site, females lose significantly more molars overall $(p=0.0088)$. Specifically, the Link/Slayden females lost $46 \%$ $(n=13 / 28)$ while males lost $13 \%(n=4 / 30)$. Antithetically, there is very little sex difference in AMTL in the Gray Farm sample. It is restricted to the incisiform teeth (I/C) with males losing significantly more (males $50 \%$ [ $n=13 / 26$ ], females $13.6 \%$ $[n=3 / 22])$. In the Thompson Village sample, first 
Table 2. Raw frequencies of antemortem tooth loss by tooth category segregated by sex and by three skeletal age-atdeath categories.

\begin{tabular}{|c|c|c|c|c|c|c|c|c|c|c|c|c|}
\hline \multirow{3}{*}{$\frac{\text { Tooth }}{\text { I/C }}$} & \multicolumn{12}{|c|}{$\begin{array}{l}\text { ANTEMORTEM TOOTH LOSS1 } \\
\text { LINK FARM/SLAYDEN }\end{array}$} \\
\hline & \multicolumn{2}{|c|}{ TOTAL } & \multicolumn{2}{|c|}{$\underline{\text { males }}$} & \multicolumn{2}{|c|}{ females } & \multicolumn{2}{|c|}{ young } & \multicolumn{2}{|c|}{$\underline{\mathrm{m} \text { aged }}$} & \multicolumn{2}{|c|}{ old } \\
\hline & $3 / 124$ & 2.4 & $0 / 17$ & 0.0 & $3 / 77$ & 3.9 & $1 / 28$ & 3.6 & $0 / 37$ & 0.0 & $2 / 10$ & 20.0 \\
\hline PM & $8 / 106$ & 7.5 & $0 / 17$ & 0.0 & $8 / 63$ & 12.7 & $4 / 16$ & 25.0 & $3 / 36$ & 8.3 & $0 / 13$ & 0.0 \\
\hline M1 & $19 / 64$ & 29.7 & $4 / 19$ & 22.2 & $5 / 29$ & 17.2 & $2 / 10$ & 20.0 & $11 / 22$ & 0.0 & $3 / 6$ & 50.0 \\
\hline M2 & $8 / 56$ & 14.3 & $2 / 18$ & 11.0 & $4 / 19$ & 21.0 & $0 / 11$ & 0.0 & $6 / 20$ & 0.0 & $0 / 4$ & 0.0 \\
\hline M3 & $8 / 41$ & 19.5 & $5 / 17$ & 29.4 & $2 / 13$ & 11.1 & $0 / 16$ & 0.0 & $0 / 26$ & 0.0 & $0 / 5$ & 0.0 \\
\hline ALL M & $35 / 161$ & 21.7 & $11 / 54$ & 20.4 & $11 / 61$ & 18.0 & $2 / 37$ & 5.4 & $17 / 58$ & 29.3 & $3 / 15$ & 33.0 \\
\hline \multirow{3}{*}{$\begin{array}{c}\text { ALL } \\
\text { DENT }\end{array}$} & $46 / 391$ & 11.8 & $11 / 88$ & 12.5 & $22 / 201$ & 10.9 & $6 / 81$ & 7.4 & $20 / 120$ & 6.7 & $5 / 38$ & 13.2 \\
\hline & \multicolumn{12}{|c|}{ GRAY FARM } \\
\hline & \multicolumn{2}{|c|}{ TOTAL } & \multicolumn{2}{|c|}{$\underline{\text { males }}$} & \multicolumn{2}{|c|}{ females } & \multicolumn{2}{|c|}{ young } & \multicolumn{2}{|c|}{$\underline{m \text { aged }}$} & \multicolumn{2}{|c|}{ old } \\
\hline $\mathrm{I} / \mathrm{C}$ & $46 / 140$ & 33.0 & $29 / 64$ & 45.3 & $8 / 59$ & 13.5 & $0 / 23$ & 0.0 & $19 / 52$ & 36.5 & $18 / 33$ & 54.5 \\
\hline PM & $38 / 124$ & 30.6 & $15 / 40$ & 37.5 & $6 / 42$ & 14.3 & $0 / 15$ & 0.0 & $22 / 45$ & 49.0 & $4 / 14$ & 28.6 \\
\hline M1 & $26 / 61$ & 42.6 & $11 / 21$ & 52.4 & $7 / 20$ & 35.0 & $0 / 8$ & 0.0 & $16 / 23$ & 9.5 & $3 / 6$ & 50.0 \\
\hline M2 & $22 / 59$ & 37.3 & $9 / 26$ & 34.6 & $7 / 20$ & 35.0 & $1 / 7$ & 14.3 & $16 / 23$ & 69.5 & $1 / 5$ & 20.0 \\
\hline M3 & $23 / 50$ & 46.0 & $10 / 19$ & 52.6 & $5 / 18$ & 27.8 & $0 / 6$ & 0.0 & $16 / 22$ & 72.7 & $2 / 7$ & 28.6 \\
\hline ALL M & $71 / 170$ & 41.8 & $30 / 66$ & 45.5 & $19 / 58$ & 32.7 & $1 / 21$ & 4.8 & $48 / 68$ & 70.6 & $6 / 18$ & 33.0 \\
\hline \multirow{3}{*}{$\begin{array}{c}\text { ALL } \\
\text { DENT }\end{array}$} & \multicolumn{2}{|c|}{$155 / 434 \quad 35.7$} & $74 / 170$ & 43.5 & $33 / 159$ & 20.8 & $1 / 59$ & 1.7 & $89 / 165$ & 4.0 & $28 / 65$ & 43.0 \\
\hline & \multicolumn{12}{|c|}{ THOMPSON } \\
\hline & \multicolumn{2}{|c|}{ TOTAL } & \multicolumn{2}{|c|}{$\underline{\text { males }}$} & \multicolumn{2}{|c|}{ females } & \multicolumn{2}{|c|}{ young } & \multicolumn{2}{|c|}{$\underline{\mathrm{m} \text { aged }}$} & \multicolumn{2}{|c|}{ old } \\
\hline $\mathrm{I} / \mathrm{C}$ & $44 / 743$ & 6.0 & $6 / 300$ & 2.0 & $24 / 348$ & 6.9 & $0 / 70$ & 0.0 & $21 / 266$ & 7.8 & $5 / 38$ & 79.0 \\
\hline PM & $49 / 554$ & 8.8 & $8 / 228$ & 3.5 & $26 / 257$ & 10.1 & $4 / 56$ & 7.1 & $26 / 189$ & 13.8 & $10 / 29$ & 34.5 \\
\hline M1 & $81 / 253$ & 32.0 & $26 / 106$ & 24.5 & $42 / 114$ & 36.8 & $6 / 28$ & 21.4 & $49 / 97$ & 24.9 & $0 / 8$ & 0.0 \\
\hline M2 & $79 / 247$ & 32.0 & $31 / 102$ & 30.4 & $37 / 113$ & 32.7 & $4 / 23$ & 17.4 & $40 / 85$ & 47.0 & $3 / 8$ & 37.5 \\
\hline M3 & $82 / 215$ & 38.1 & $32 / 83$ & 38.5 & $37 / 97$ & 38.1 & $5 / 24$ & 20.8 & $43 / 78$ & 55.1 & $1 / 7$ & 14.3 \\
\hline ALL M & $142 / 715$ & $5 \quad 33.8$ & $89 / 291$ & 30.6 & $116 / 324$ & 35.8 & $15 / 75$ & 20.0 & $132 / 26$ & $\begin{array}{ll}0 & 0.8\end{array}$ & $4 / 23$ & 17.4 \\
\hline ALL & $235 / 201$ & 1211.7 & $103 / 81$ & 912.6 & $166 / 929$ & 17.9 & $25 / 20$ & 112.4 & $179 / 71$ & 525.0 & $19 / 90$ & 21.1 \\
\hline & & & & & & & & & & & & \\
\hline & $\underline{\text { TOT }}$ & TAL & $\underline{\mathrm{ma}}$ & & fem & tales & & oung & $\underline{\mathrm{ma}}$ & ged & & ld \\
\hline $\mathrm{I} / \mathrm{C}$ & $1 / 55$ & 1.8 & $0 / 23$ & 0.0 & $1 / 32$ & 3.1 & $0 / 8$ & 0.0 & $0 / 21$ & 0.0 & $1 / 10$ & 10.0 \\
\hline PM & $4 / 35$ & 11.4 & $0 / 6$ & 0.0 & $4 / 13$ & 30.1 & $0 / 6$ & 0.0 & $0 / 15$ & 0.0 & $4 / 7$ & 57.1 \\
\hline M1 & $4 / 20$ & 20.0 & $0 / 2$ & 0.0 & $4 / 6$ & 66.0 & $0 / 2$ & 0.0 & $1 / 13$ & 7.7 & $3 / 3$ & 100.0 \\
\hline M2 & $3 / 24$ & 12.5 & $1 / 5$ & 20.0 & $2 / 17$ & 11.8 & $0 / 3$ & 0.0 & $0 / 10$ & 0.0 & $3 / 3$ & 100.0 \\
\hline M3 & $2 / 19$ & 10.5 & $0 / 1$ & --- & $2 / 5$ & 40.0 & $0 / 2$ & 0.0 & $0 / 8$ & 0.0 & $2 / 3$ & 67.0 \\
\hline ALL M & $9 / 63$ & 14.3 & $1 / 8$ & 12.5 & $8 / 28$ & 28.6 & $0 / 7$ & 0.0 & $0 / 31$ & 0.0 & $9 / 9$ & 100.0 \\
\hline $\begin{array}{c}\text { ALL } \\
\text { DENT }\end{array}$ & $14 / 153$ & 9.1 & $1 / 37$ & 2.7 & $13 / 73$ & 17.8 & $0 / 21$ & 0.0 & $1 / 67$ & 1.5 & $13 / 26$ & 50.0 \\
\hline
\end{tabular}

${ }^{1}$ Dentition was segregated by sex or age; sex-indeterminate dentition was included in the age-at-death samples. 
Table 3. Pair-wise statistical comparisons of antemortem tooth loss based on the raw data from Table1. No tests were undertaken if a sample was less than ten cases. Statistical significance $(p=\leq 0.05)$ is indicated by bold font.

\begin{tabular}{|c|c|c|c|c|c|c|}
\hline & $\begin{array}{l}\text { Link/Slayden } \\
\text { x Gray Farm }\end{array}$ & $\begin{array}{l}\text { Link/Slayden } \\
\text { x Thompson }\end{array}$ & $\begin{array}{l}\text { Gray Farm } \\
\text { x Thompson }\end{array}$ & $\begin{array}{c}\text { Gray Farm } \\
\text { x Hobbs }\end{array}$ & $\begin{array}{c}\text { Thompson } \\
\text { x Hobbs }\end{array}$ & $\begin{array}{c}\text { Link/Slayden } \\
\text { x Hobbs }\end{array}$ \\
\hline \multicolumn{7}{|c|}{ All } \\
\hline I/C & 0.0001 & 0.0093 & 0.0001 & 0.0001 & 0.3572 & 1.0000 \\
\hline PM & 0.0001 & 0.5190 & 0.0001 & 0.0288 & 0.5440 & 0.3119 \\
\hline M1 & 0.1413 & 0.0592 & 0.1329 & 0.1083 & 0.3236 & 0.5672 \\
\hline M2 & 0.0196 & 0.0234 & 0.4444 & 0.0342 & 0.0611 & 0.7586 \\
\hline M3 & 0.0428 & 0.0908 & 0.3373 & 0.0103 & 0.0222 & 0.2130 \\
\hline All M & 0.0001 & 0.0005 & 0.0607 & 0.0001 & 0.0001 & 0.2632 \\
\hline All Dent & 0.0001 & 0.0001 & 0.0001 & 0.0001 & 0.0160 & 0.4479 \\
\hline \multicolumn{7}{|c|}{ Young } \\
\hline $\mathrm{I} / \mathrm{C}$ & 1.0000 & 0.3519 & 0.4017 & ---- & ---- & ---- \\
\hline PM & 0.3094 & 0.6704 & 0.6490 & --- & --- & --- \\
\hline M1 & ---- & 1.0000 & ---- & --- & --- & ---- \\
\hline M2 & ---- & 0.2741 & ---- & ---- & --- & ---- \\
\hline M3 & ---- & 0.0712 & ---- & ---- & ---- & ---- \\
\hline All M & 0.6495 & 0.1040 & 0.0156 & ---- & ---- & ---- \\
\hline All Dent & 0.2239 & 0.1681 & 0.0044 & 1.0000 & 0.1408 & 0.3410 \\
\hline \multicolumn{7}{|c|}{ Middle Age } \\
\hline $\mathrm{I} / \mathrm{C}$ & 0.0001 & 0.0887 & 0.0001 & 0.0008 & 0.3810 & 1.0000 \\
\hline PM & 0.0001 & 0.5865 & 0.0001 & 0.0004 & 0.2255 & 0.5462 \\
\hline M1 & 0.2307 & 1.0000 & 0.1100 & 0.0004 & 0.0056 & 0.0132 \\
\hline M2 & 0.0148 & 0.2136 & 0.0636 & 0.0003 & 0.0045 & 0.0741 \\
\hline M3 & 0.0001 & 0.0001 & 0.1515 & --- & --- & --- \\
\hline All M & 0.0001 & 0.0037 & 0.0039 & 0.0001 & 0.0001 & 0.0004 \\
\hline All Dent & 0.0001 & 0.4091 & 0.0001 & 0.0001 & 0.0001 & 0.0012 \\
\hline \multicolumn{7}{|c|}{ Old Age } \\
\hline $\mathrm{I} / \mathrm{C}$ & 0.0764 & 0.6251 & 0.0003 & 0.0261 & 1.0000 & 1.0000 \\
\hline PM & 0.0978 & 0.0182 & 1.0000 & ---- & ---- & --- \\
\hline M1 & ---- & --- & ---- & --- & --- & --- \\
\hline M2 & --- & ---- & ---- & ---- & ---- & ---- \\
\hline M3 & ---- & --- & ---- & ---- & ---- & ---- \\
\hline All M & 0.0135 & 0.0002 & 0.2889 & ---- & --- & ---- \\
\hline All Dent & 0.6781 & 0.0780 & 0.0045 & 0.3643 & 0.5953 & 0.2073 \\
\hline
\end{tabular}

${ }^{1}$ Fisher's exact test, $p=\leq 0.05$; sample $\mathrm{n}=\geq 10$

molars and molars collectively are more commonly sex between the sites (Table 6). Comparing the lost by females. Females lost $\sim 57 \%$ of M1 $(\mathrm{n}=29 / 51)$ compared to $34 \%(\mathrm{n}=18 / 53)$ in the males. The disparity drives the significance for all molars $(p=0.0272)$ and collectively for all dentition (males $\mathrm{n}=67 / 397$ [16.9\%], females $\mathrm{n}=97 / 405$ [24\%]).

Since the three sites with testable samples may have samples that may have a temporal bias (e.g., Link/Slayden biased by some temporally later Link cases), it is possible that the AMTL varies by larger Middle Age category by sex and by site, females apparently vary very little between samples. Thompson females significantly differ from Gray Farm females for only the premolars (PM) $(\mathrm{p}=$ 0.0382, Thompson 10.6\% [n=12/113], Gray Farm $31.2 \%[n=5 / 16])$. There are also no statistical differences when all molars are pooled $(p=0.0727)$ (Thompson 53\% [n=77/145], Gray Farm 41\% $[n=25 / 61])$. The scenario is different for the males. The Link/Slayden sample, with overall the fewest 
teeth lost antemortem (7.7\%), has fewer molar tooth loss than Thompson $(p=0.0426)$ and exhibits significantly fewer tooth loss in all tooth categories from the Gray Farm sample (with a total tooth loss of $77.8 \%$ ). Although both Gray Farm and Thompson village were occupied until regional abandonment, the Thompson site sample has a temporally later sample bias than Gray Farm. Thompson Village males have significantly fewer AMTL in all but third molar loss (see Table 5). Overall tooth loss for Thompson males is $16.9 \%$.

\section{Discussion}

Although etiologically multifactorial, the primary clinical causes of AMTL are the progressive pathol- ogies of caries and periodontal disease (e.g., Baelum et al., 1986; Kida et al., 2006; Müller \& Hussein, 2017; Niessen \& Weyant, 1989; Ong, 1998; van der Velden et al., 2015). Both processes are associated with the consumption of carbohydrates in combination with poor oral hygiene (e.g., Baumgartner et al., 2009; Chapple et al., 2017; Hix \& O'Leary, 1976). As a proxy for pre-Columbian carbohydrate consumption, AMTL has the potential to augment archaeological information about diet and subsistence. In the context of the preColumbian skeletal samples from the Kentucky Lake Reservoir of northern west-central Tennessee, the insights are more than supplemental as archaeological assessment is limited and will likely con-

Table 4. Comparisons of antemortem tooth loss by sex for each site. No test was undertaken if either the male or female cohort consisted of less than ten cases. Statistical significance $(p=\leq 0.05)$ is indicated by bold font.

\begin{tabular}{|c|c|c|c|c|}
\hline & Link/Slayden & Thompson & Gray Farm & Hobbs \\
\hline \multicolumn{5}{|c|}{ All Adults } \\
\hline $\mathrm{I} / \mathrm{C}$ & 1.0000 & 0.0042 & 0.0002 & 1.0000 \\
\hline PM & 1.0000 & 0.0043 & 0.0225 & 0.2554 \\
\hline M1 & 1.0000 & 0.0579 & 0.3499 & ---- \\
\hline M2 & 0.7057 & 0.7697 & 1.0000 & 1.0000 \\
\hline M3 & 0.3892 & 1.0000 & 0.1837 & ---- \\
\hline All M & 0.3981 & 0.0827 & 0.1976 & 0.6478 \\
\hline All Dent & 0.6908 & 0.0022 & 0.0001 & 0.0851 \\
\hline \multicolumn{5}{|c|}{ Young } \\
\hline $\mathrm{I} / \mathrm{C}$ & --- & 0.5762 & 1.0000 & --- \\
\hline PM & ---- & 1.0000 & 0.2308 & --- \\
\hline M1 & --- & 1.0000 & --- & --- \\
\hline M2 & --- & 1.0000 & --- & ---- \\
\hline M3 & -.-- & 0.4423 & ---- & ----- \\
\hline All M & 0.2881 & 1.0000 & 0.0420 & --- \\
\hline All Dent & 0.5918 & 0.5810 & 0.0223 & ---- \\
\hline \multicolumn{5}{|c|}{ Middle Age } \\
\hline $\mathrm{I} / \mathrm{C}$ & 1.0000 & 0.7854 & 0.0131 & --- \\
\hline PM & 0.3416 & 0.8239 & 0.5166 & --- \\
\hline M1 & --- & 0.0297 & 1.0000 & ---- \\
\hline M2 & --- & 0.0656 & 1.0000 & ---- \\
\hline M3 & 1.0000 & 0.5169 & 1.0000 & ----- \\
\hline All M & 1.0000 & 0.0272 & 1.0000 & ---- \\
\hline All Dent & 1.0000 & 0.0001 & 0.0646 & ---- \\
\hline \multicolumn{5}{|c|}{ Old Age } \\
\hline I/C & ---- & 0.1469 & --- & --- \\
\hline PM & ---- & 0.0061 & --- & ---- \\
\hline M1 & ---- & 0.1939 & - & --- \\
\hline M2 & ---- & 0.2364 & --- & ---- \\
\hline M3 & ---- & 0.1939 & - & --- \\
\hline All M & 0.1923 & 0.0021 & --- & ---- \\
\hline All Dent & 0.6882 & 0.0008 & --- & --- \\
\hline
\end{tabular}


Table 5. The Middle Age category generated the largest samples of antemortem tooth loss segregable by sex. However, several tooth classes generated samples of less than ten teeth. These were not eligible for statistical comparisons.

\begin{tabular}{|c|c|c|c|c|c|c|}
\hline & \multicolumn{2}{|c|}{ Link/Slayden } & \multicolumn{2}{|c|}{ Gray Farm } & \multicolumn{2}{|c|}{ Thompson } \\
\hline & \multicolumn{6}{|c|}{ MA Males } \\
\hline $\mathrm{I} / \mathrm{C}$ & $0 / 12$ & $0.0 \%$ & $13 / 26$ & $50.0 \%$ & $6 / 146$ & $4.6 \%$ \\
\hline PM & $0 / 10$ & $0.0 \%$ & $10 / 23$ & $43.5 \%$ & $10 / 108$ & $9.3 \%$ \\
\hline M1 & $3 / 10$ & $30.0 \%$ & $9 / 12$ & $75.0 \%$ & $18 / 53$ & $34.0 \%$ \\
\hline M2 & $1 / 10$ & $10.0 \%$ & $8 / 12$ & $67.0 \%$ & $15 / 50$ & $30.0 \%$ \\
\hline M3 & $0 / 10$ & $0.0 \%$ & $9 / 12$ & $75.0 \%$ & $18 / 40$ & $45.0 \%$ \\
\hline All M & $4 / 30$ & $13.3 \%$ & $26 / 36$ & $72.2 \%$ & $51 / 143$ & $35.7 \%$ \\
\hline \multirow[t]{2}{*}{ All Dent } & $4 / 52$ & $7.7 \%$ & $49 / 63$ & $77.8 \%$ & $67 / 397$ & $16.9 \%$ \\
\hline & \multicolumn{6}{|c|}{ MA Females } \\
\hline $\mathrm{I} / \mathrm{C}$ & $0 / 25$ & $0.0 \%$ & $3 / 22$ & $13.6 \%$ & $8 / 147$ & $54.4 \%$ \\
\hline PM & $3 / 16$ & $18.8 \%$ & $5 / 16$ & $31.3 \%$ & $12 / 113$ & $10.6 \%$ \\
\hline M1 & $8 / 12$ & $67.0 \%$ & $6 / 8$ & $75.0 \%$ & $29 / 51$ & $56.9 \%$ \\
\hline M2 & $5 / 10$ & $50.0 \%$ & $6 / 8$ & $75.0 \%$ & $24 / 49$ & $49.9 \%$ \\
\hline M3 & $0 / 6$ & $0.0 \%$ & $5 / 7$ & $71.4 \%$ & $24 / 45$ & $53.3 \%$ \\
\hline All M & $13 / 28$ & $46.4 \%$ & $17 / 23$ & $73.9 \%$ & $77 / 145$ & $53.1 \%$ \\
\hline All Dent & $16 / 68$ & $23.5 \%$ & $25 / 61$ & $41.0 \%$ & $97 / 405$ & $24.0 \%$ \\
\hline
\end{tabular}

${ }^{1}$ statistical tests not undertaken if sample size $\leq 10$
Table 6. For samples of at least ten individuals, statistical comparisons were undertaken between the site samples by sex. Statistical significance $(p=\leq 0.05)$ is indicated by bold font.

\begin{tabular}{lccc}
\hline & $\begin{array}{c}\text { Link/Slayden } \mathrm{v} \\
\text { Thompson }\end{array}$ & $\begin{array}{c}\text { Link Slayden v } \\
\text { Gray Farm }\end{array}$ & $\begin{array}{c}\text { Thompson v } \\
\text { Gray Farm }\end{array}$ \\
\hline I/C & 1.0000 & Males & \\
PM & 1.0000 & $\mathbf{0 . 0 0 2 6}$ & $\mathbf{0 . 0 0 0 1}$ \\
M1 & 1.0000 & $\mathbf{0 . 0 3 0 3}$ & $\mathbf{0 . 0 0 0 3}$ \\
M2 & 0.2629 & $\mathbf{0 . 0 2 3 7}$ & $\mathbf{0 . 0 2 8 7}$ \\
M3 & $\mathbf{0 . 0 0 8 5}$ & $\mathbf{0 . 0 1 1 5}$ & $\mathbf{0 . 0 4 2 2}$ \\
All M & $\mathbf{0 . 0 4 2 6}$ & $\mathbf{0 . 0 0 1 6}$ & 0.2021 \\
All Dent & 0.1473 & $\mathbf{0 . 0 0 0 1}$ & $\mathbf{0 . 0 0 0 1}$ \\
\hline & & $\mathbf{0 . 0 0 0 1}$ & $\mathbf{0 . 0 0 0 1}$ \\
\hline I/C & 0.6048 & Females & \\
PM & 0.3986 & 0.0950 & 0.1577 \\
M1 & 0.7462 & 0.6851 & $\mathbf{0 . 0 3 8 2}$ \\
M2 & 1.0000 & ---- & --- \\
M3 & ---- & ---- & -- \\
All M & 0.5421 & ---- & 0.0727 \\
All Dent & 0.3063 & 0.0858 & 0.0601 \\
\hline
\end{tabular}

${ }^{1}$ Tests undertaken on samples $\geq 10$ tinue to be so for the foreseeable future. Absent from the KLR archaeological record are radiocarbon dates, therefore, using attributes of the material culture, the sites have been temporally sequenced relative to each other (Bass, 1985; Dye 2002, 2003, 2004; Lunn, 2013). Pairing the occupation sequence with the AMTL data in the Kentucky Lake Reservoir yielded several potentially interpretively valuable patterns. Unfortunately, poor sample sizes restrict assessments by skeletal age at death. Therefore, the interpretations of the rate and pattern of AMTL should be considered tentative.

\section{Temporal pattern}

Hobbs, the earliest site sample evaluated here, dates to the Late Woodland period ( AD 500-900). Overall, there are fewer teeth lost antemortem by the Hobbs mortuary site relative to the Middle-toLate Middle Mississippian Period Gray Farm and Thompson Village samples. The frequencies are consistent with the reconstruction of Late Woodland subsistence in the Tennessee River Valley as pre-maize horticulturalists with (at least) seasonal foraging (e.g., mast, fleshy fruits) (Bense, 2016; Crites, 1978; Emerson et al., 2000; Kline et al., 1982; McMahan, 1983). Cultigens utilized were the grasses native to the local environment. These were the oily (e.g., Iva [sumpweed], Helianthus [sunflower]) and starchy seeds (e.g, Chenopodium [goosefoot],
Phalaris [maygrass], Polygonum [knotweed], Hordeum [little barley]), the latter of which are cariogenic and capable of initiation and progression of tooth decay (e.g., Lingström et al., 2000; Pollard, 1995). The AMTL results for Hobbs parallels the prevalence of caries (Smith \& Betsinger, 2019). That is, there were significantly fewer carious teeth compared to maize-intensive samples.

The small Hobbs sample size did not permit age -at-death AMTL comparisons. Indeed, the sample was biased in favor of older individuals which, given the progressive nature of AMTL, might bias in favor of congruence with samples archaeologically identified as agriculturalist. However, when segregated by age-at-death in the larger Middle Age category, Hobbs sustains the pattern of significantly fewer AMTL for all tooth categories compared to Gray Farm and for the posterior teeth in Thompson.

The AMTL of Link/Slayden relative to Hobbs Archaeologically, the Link/Slayden polity exhibits the material culture attributes of the Mississippian period (Bass, 1985; Dye, 2002, 2003, 2013; Lunn, 2013). The Link site (AD 1250-1400) is a civicceremonial mound center complex with a central plaza (Bass, 1985; Dye, 2007, 2012; Nash, 1968). Although Bass (1985) characterized Slayden as an expansion site from Link, recent ceramic analysis 
suggests it is the earlier (circa AD 1050-1250) of the two (Dye, 2004; Lunn, 2013). The Link site was arguably abruptly abandoned circa AD 1400, based on the archaeological context of the Duck River Cache (Dye, 2007, 2012; Nash, 1968), a large assemblage of ritual performance objects (e.g., crownform clubs, monolithic axes, raptor talon claw effigies) recovered from the site. The cache was arguably deliberately buried as a last act prior to (possibly warfare-related) site abandonment (Dye, 2007; Dye \& King, 2007). Although there is a general congruence of AMTL between the Link/ Slayden sample and the Late Woodland Hobbs sample (see Table 2), when the samples are segregated by age at death, the Mississippian Link/ Slayden sample does exhibit more molar loss, particularly M1, the molar longest in occlusion. The M1 sample size drives the difference in all molars and all the dentition.

Previous assessment of the caries pattern and prevalence in the Hobbs sample indicated an overall higher prevalence of carious teeth compared to the Link/Slayden sample (although few tooth classes are significantly higher) (Smith \& Betsinger, 2019). Considering the temporal and archaeological context of Link/Slayden, maize was unequivocally available as a productive cultigen. It is possible that as a shortfall-hedging strategy, food production within the Link polity included seasonal foraging and/or cultivation of native cultigens (e.g., Gremillion et al., 2008). It is also possible that the WPA era (Works Progress Administration, 1939-1943) (Dye, 2017) salvage recovery protocols at Link/Slayden biased in the direction of a temporally earlier (i.e., incipient/early maize adoption) mortuary sample. More speculative, but an aspect to consider given the archaeologically-based conclusion by Bass (1985) that the Link polity is ethnically different from Gray Farm, is regional variability in the symbolic or ritual role of maize or, in the routine maize processing and/or preparation (e.g., roasted, hominy, hoecake, and/or corn mush) which may have reduced the oral bioavailability of starch (e.g., Blitz, 1993; Briggs, 2016; Fritz \& Lopincot, 2007; Katz et al., 1974; Peres, 2017; Raviele, 2011).

\section{The AMTL differences between the Mississippian} samples

In the large Middle Age cohort, the Link/Slayden site sample has significantly fewer AMTL than Gray Farm in all tooth categories except the first molars. This is unexpected given the apparently extensive temporal overlap of the sites, but may be explained by the scenarios presented above. The Gray Farm (AD 1150-1400) site sample might be considered exemplary of maize-intensive Mississippian agriculturalization. Based on the prevalence of carious teeth by tooth type and age-atdeath, the Gray Farm dental sample certainly allies with definitively maize-intensive Late Mississippian period sites from (at least) East Tennessee whereas Link/Slayden does not (Smith \& Betsinger, 2019). However, in the Middle Age cohort, compared to the maize-intensive samples, the Gray Farm dental sample exhibits small lesion size and virtually no cases of pulp exposure. If the progression of tooth decay was comparatively rapid in the Gray Farm sample, the carious lesions achieving pulp penetration may have precipitated the earlier exfoliation of affected teeth, generating the high AMTL results seen here. The co-occurrence of larger lesions with fewer teeth lost antemortem can potentially be tested in the Late Mississippian period sites from East Tennessee. This faster rate could also explain the significant increase in the frequency of AMTL in the anterior teeth (I/C, PM) of Gray Farm relative to both Link/Slayden and Thompson Village (see Table 2). Caries and AMTL initially progress in the complex-crowned teeth with greater interstitial surface area (e.g., Broadbent et al., 2006; Carlos \& Gittelsohn, 1965). The greater involvement of the anterior teeth in Gray Farm may signal an oral environment of advanced tooth decay (e.g., O'Sullivan \& Tinanoff, 1993). Possible mitigating factors, as yet unknown, are dental issues such as LEH which might predispose the incisiform teeth to pulp-penetrating demineralization.

It can be assumed that maize was the primary cariogenic carbohydrate consumed by the community residing in the Gray Farm site. But evidently, the consumption was differential by, at least, sex (see Table 5), but not by the same sex. In the Middle Age category, Gray Farm males exhibit more AMTL than females (77.8 percent versus 41.0 percent) while the females of Link/Slayden have the higher frequency (23.5 percent versus 7.7 percent). Pregnant and nursing females are arguably more vulnerable to tooth decay and in archaeological contexts may signal reproductive stress (Ferraro and Vieira, 2010; Lukacs, 2011; Lukacs and Largaespada, 2006; Walter et al., 2016), but the statistically significant higher AMTL among females in the Link/Slayden sample $(p=0.0260)$ may also suggest differential consumption of decay-causing carbohydrates (e.g., Larsen, 1983; Somerville et al., 2015). Greater maize consumption by females has been documented elsewhere in the Mississippian 
world (Ambrose et al., 2003; Larsen, 1983). The higher rate of AMTL in the males of Gray Farm strongly suggests greater consumption. This is underscored by the between sample comparison by sex in the larger Middle Age category (see Tables 4 and 5). There are few differences in the prevalence of AMTL among the females for all tooth types and all teeth between Link/Slayden, Gray Farm, and Thompson Village. However, male AMTL is significantly higher in Gray Farm than either

Link/Slayden or Thompson Village in the vast majority of statistical comparisons. The reason for this pattern might include the use of maize in malepredominated ritual feasting. Given the similarity of female AMTL across the site samples, it does not suggest a simple community pattern of differential maize dietary consumption. As stated earlier, Bass (1985) has argued that the Link and Gray Farm polities are ethnically different. This is based on the differences in mortuary protocols in addition to the considerable geographic distance between the two polities. This is certainly plausible given the large buffer zone between the polities in addition to an intervening polity with its own large mound center (Dye, 2004). However, the high frequency and male bias of AMTL is not repeated in the Thompson Village sample, a satellite community within the Gray Farm polity.

Based on the ceramic sequence and temporal pattern of domestic structure construction, the Gray Farm site was occupied earlier than the Thompson site with the founding of the Williams site (40HY1) (no AMTL data), part of the Gray Farm polity, straddling the two (Bass, 1985). Gray Farm, therefore, would have had a mortuary component earlier in time than Thompson Village. Both the Gray Farm and Thompson Village sites generated Late Mississippian period (post AD 1300) ceramic types which suggests both sites, as well as Link, were occupied until regional abandonment ("Vacant Quarter") of the $14^{\text {th }}$ and $15^{\text {th }}$ centuries (Cobb \& Butler, 2002). If maize adoption intensified with Mississippianization, as conventional wisdom suggests (Bense, 2016; Emerson et al., 2020), the Thompson AMTL frequencies for all tooth types should be greater than Gray Farm, even if not significantly so (see Tables 1 and 2). They are not. The results may be a by-product of sampling error (biased site recovery, differential preservation, and/or small sample sizes) or reflect local socioeconomic issues. Thompson Village was a satellite community within the Gray Farm polity and may not have engaged in, or had access to, the same civic-ceremonial activities of a mound center.
That is, if indeed the male AMTL bias at Gray Farm is reflective of non-dietary maize consumption. But the circumstance preceding regional abandonment may have also been a factor in the apparent better oral health of the temporally later Thompson Village sample.

\section{Climate volatility}

Maize cultivation may have been affected by a series of droughts that occurred in the Southeast beginning circa AD 1100 (Aharon et al., 2012; Benson et al., 2009) and in Tennessee in the Middle Cumberland River culture area (Figure 1) circa AD 1288 -1388 (Meeks \& Anderson, 2013). Agricultural shortfall may have episodically reduced overall carbohydrate consumption potentially causing nutritional stress or dietary shifts to foraged resources (e.g., Scopa Kelso, 2018). This may have been reflective of, or exacerbated by, the onset after AD 1300 of the climate phenomenon of the Little Ice Age (Bird et al., 2017; Naftz et al., 1996; Stahle \& Cleaveland, 1994; Wilson, 2017). Nutritional stress may not be documentable, ${ }^{1}$ but dietary shifts are certainly a plausible scenario based on (for example) the botanical evidence from the Illinois River Valley of the apparent juxtaposition of maize adoption and reduction in arboreal seed crop harvest (VanDerwarker et al., 2017). Mast and fleshy fruits may have been fall-back options in times of carbohydrate harvest insufficiency. This may be what was occurring in the Link polity and in the later occupation within the Gray Farm polity.

\section{Political instability}

Perhaps synergistically related to climate change, there is a wide-spread decline of polities in the late Mississippian period (after AD 1300) (Anderson, 1994, 1996; Benson, et al., 2009; Bird et al., 2017; Wilson, 2017). Although particular mound centers as loci of civic and ceremonial functions did fluctuate over time and space (e.g., Anderson, 1996; Beck, 2003; Benson et al., 2009; Blitz, 1999; Wilson, 2017), in the Middle Cumberland River Valley to the East of the KLR samples, political destabilization occurs circa AD 1325 (Dye, 2004; Krus \& Cobb, 2015; Vidoli, 2012) just prior to the regional abandonment. This is archaeologically manifested by the reduction in centralized authority and increase in settlement fortification (palisades) (Krus \& Cobb, 2015). Warfare is argued to escalate during this time period and, in the KLR, the apparent abrupt abandonment of the Link site (i.e., burial of the Duck River Cache) has been attributed to imminent intergroup violence (Dye, 2004; Dye \& 
King, 2007). Bass argued that it is only in the later occupation sequence of the Mississippian sites that palisades are firmly documented (1985:216). Corroboratively, based on a shift in mortuary patterning, Bass argues the changes "indicate that the sociopolitical structure of these polities in the late Middle Mississippian Period... had altered to ones in which elite status or high rank was truncated and invested in and restricted to only one rank, an individual kin group or family which produced the chiefly line (1985:195)." Perhaps the absence of male-focused decay-inducing carbohydrate consumption in the Thompson Village sample compared to Gray Farm is reflective of this political decline with an overall reduction in AMTL in Thompson is suggestive of agricultural shortfall.

\section{Conclusions}

The trend towards maize intensification cooccurring with sociopolitical complexity is a general pattern within the Mississippian period (AD 1000-1550) in what is now the lower Midwest and much of the southeastern United States. However, the transition varies temporally and unevenly between sociopolitical and/or ecological contexts. Mississippianization abruptly ended in northern west-central Tennessee by AD 1450. Occupation of the area was abandoned as part of a much broader inexplicable depopulation of the lower Ohio River Valley inclusive of the lower Tennessee River Valley. The dearth of archaeological context for the Kentucky Lake Reservoir region of northern westcentral Tennessee contributes to the conundrum of the apparent abandonment. Much basic subsistence information is archaeologically unclear but can be remediated by certain lines of bioarchaeological inquiry.

Although AMTL has a complex etiology, it has been an effective proxy for oral decay which has been bioarchaeologically observed to vary by subsistence strategy and the dietary dependence on starchy cultigens. The AMTL observed in the Kentucky Lake Reservoir yielded three patterns which segregate the site samples of Hobbs, Link/Slayden, Gray Farm and Thompson Village by temporal period and geographic distribution. The first pattern is the retention of a general Late Woodland (AD 600-900) AMTL loss (Hobbs site sample) in the Mississippian period Link polity (Link/Slayden site sample). The pattern does not indicate a maizeintensive subsistence strategy. It may reflect a strategically mitigated economic adjustment to include foraged resources consequential to demonstrable climate volatility ( AD 1400-1700). This subsistence strategy is similar to what has been archaeo- logically reconstructed for the pre-maize Late Woodland period. However, this pattern may also reflect possible salvage archaeology recovery bias inadvertently favoring temporally earlier Link/ Slayden burials, which may indeed have practiced a Late Woodland subsistence economy. Less likely, but culturally plausible given that Bass argued ethnic differences between the Link and Gray Farm polities, there may have been polity-variable food preparation techniques which reduce the opportunities for dental decay in the oral environment.

The second pattern is a regional difference in Mississippian period maize adoption. The caries data as well as AMTL of the Gray Farm site sample is consistent with maize-intensive agriculturalization and in contrast with the lower prevalence results of the Link polity. Indeed, AMTL is higher than the Thompson Village site sample, a temporally later-founded satellite community of the Gray Farm polity. It is possible that Gray Farm reflects a temporal window of successful maize overcrop which then declined just prior to regional abandonment. Alternatively, maize may have been siphoned from satellite communities to the Gray Farm site as it was the administrative capitol for the polity.

The third pattern is the higher overall prevalence of AMTL in the males of the Gray Farm sample. This contrasts with the Link/Slayden and Thompson Village samples which indicated more AMTL among the females. Interpretively important, the prevalence of AMTL is not significantly different between the females in the three Mississippian period site samples. Therefore, the Gray Farm male pattern may reflect specific malefocused civic-ceremonial roles which involved maize consumption that may have declined in the later Thompson Village context paralleling documented sociopolitical decline. Alternatively, the civic-ceremonial activity may not have been undertaken in satellite communities.

The patterns apparent in this study may be a consequence of sampling error from possible excavation bias as well as small sample sizes available for assessment. However, the patterns may indeed reflect the political landscape as well as the climate, ecological, and sociopolitical challenges of the thirteenth century prior to regional abandonment. Hopefully, more health status data can be marshaled to clarify the regional and temporal patterns.

${ }^{1}$ Access to the osteological collections has now been terminated pending reburial 


\section{Acknowledgements}

The authors greatly appreciate the permissions to access and assess the collections curated by the Frank H. McClung Museum, Knoxville, Tennessee. We thank the staff for their forbearance to our presence, particularly Dr. Jefferson Chapman (Executive Director, retired), Dr. Lynne P. Sullivan (Curator of Archeology, retired), and Dr. Timothy Baumann (Curator of Archaeology). We also appreciate and commend the collecting assistance of Ms. Germaine Mosher and Ms. Amanda Bailey.

\section{REFERENCES}

Aharon, P., Aldridge, D., \& Hellstrom, J. (2012). Rainfall variability and the rise and collapse of the Mississippian Chiefdoms: Evidence from a DeSoto Caverns Stalagmite. In L. Giosan, D.Q. Fuller, K. Nicoll, R.K. Flad, \& PD Clift (Eds.), Climates, landscapes, and civilizations. Geophysical Monograph Series 198 (pp. 35-142). New York: Wiley.

Ambrose, S. H., Buikstra, J.E., \& Krueger, H.W. (2003). Status and gender differences in diet at Mound 72, Cahokia, revealed by isotopic analysis of bone. Journal of Anthropological Archaeolo$g y, 22(3), 217-226$.

Anderson, D. G. (1996). Chiefly cycling and largescale abandonments as viewed from the Savannah River Basin. In J. F. Scarry (Ed.), Political structure and change in the prehistoric Southeastern United States (pp. 150-191). Gainesville: University Press of Florida.

Baelum, V. \& Fejerskov, O. (1986). Tooth loss as related to dental caries and periodontal breakdown in adult Tanzanians. Community Dentistry and Oral Epidemiology, 14(6), 353-357.

Bardolph, D.N. (2014). Evaluating Cahokian contact and Mississippian identity politics in the late prehistoric Central Illinois River Valley. American Antiquity, 79(1), 69-89.

Bass, Q.R., II. (1985). Sociopolitical and economic aspects of the Mississippian occupation in the Lower Tennessee River Valley. Manuscript on file, Department of Sociology and Anthropology, Middle Tennessee State University, Murfreesboro.

Beahm, E.L. (2013). Mississippian polities in the Middle Cumberland region of Tennessee (Unpublished doctoral dissertation). University of Georgia, Athens, GA.

Beck, R. (2003). Consolidation and hierarchy: Chiefdom variability in the Mississippian Southeast. American Antiquity, 68(4), 641-661.

Benson, L.V., Pauketat, T.R., \& Cook, E.R. (2009). Cahokia's Boom and Bust in the Context of Cli- mate Change. American Antiquity, 74(3), 467483.

Baumgartner, S., Imfeld, T., Schicht, O., Rath, C., Persson, R.E., \& Persson, G.R. (2009). The impact of the stone age diet on gingival conditions in the absence of oral hygiene. Journal of Periodontology, 80(5), 759-768.

Bense, J.A. (2016). Archaeology of the Southeastern United States: Paleoindian to World War I. New York, NY: Routledge.

Bibby, B.G., Goldberg, H.J.V., \& Chen, E. (1951) Evaluation of caries-producing potentialities of various foodstuffs. The Journal of the American Dental Association, 42(5), 491-509.

Bird, B.W., Wilson, J.J., Gilhooly III, W.P., Steinman, B.A. \& Stamps, L. (2017). Midcontinental Native American population dynamics and late Holocene hydroclimate extremes. Scientific Reports, 7, 41628.

Betsinger, T.K., \& Smith, M.O. (2018). Regional differences in caries by sex and social status in late prehistoric East Tennessee. In S.C. Hodge, K. Schuler (Eds.), Bioarchaeology of the American Southeast: Approaches to bridging health and identity in the past (pp. 54-68). Tuscaloosa: University of Alabama Press.

Blitz, J.H. (1993) Big pots for big shots: Feasting and storage in a Mississippian community. American Antiquity, 58(1), 80-96.

Blitz, J.H. (1999). Mississippian chiefdoms and the fission-fusion process. American Antiquity, 64(4), 577-592.

Briggs, R.V. (2016) The civil cooking pot: hominy and the Mississippian standard jar in the Black Warrior Valley, Alabama. American Antiquity, 81 (2), 316-332. 1

Broadbent, J.M., Thomson, W.M., \& Poulton, R. (2006). Progression of dental caries and tooth loss between the third and fourth decades of life: a birth cohort study. Caries Research, 40(6), 459-465.

Buikstra, J.E., \& Ubelaker, D.H. (1994). Standards for data collection from human skeletal remains. Arkansas Archaeological Survey Research Series.

Carlos, J. P., \& Gittelsohn, A. M. (1965). Longitudinal studies of the natural history of caries - II: A life-table study of caries incidence in the permanent teeth. Archives of Oral Biology, 10(5), 739751.

Chapple, I.L.C., Bouchard, P., Cagetti, M.G., Campus, G., Carra, M-C., Cocco, F., \& Nibali, L. Hujoel, P., Laine, M.L., Lingström, P., Manton, D.J., Montero, E., Pitts, N., Rangé H., Schleuter N., Teughels, W., Twetman, S., Van Loveren C., 
Van der Weijden, F., Vieira, A.R., \& Schulte, A.G. (2017). Interaction of lifestyle, behaviour or systemic diseases with dental caries and periodontal diseases: consensus report of group 2 of the joint EFP/ORCA workshop on the boundaries between caries and periodontal diseases. Journal of Clinical Periodontology, 44(18), S39-S51.

Cobb, C.R. (2003). Mississippian chiefdoms: How complex? Annual Review of Anthropology, 32(1), 63-84.

Cobb, C.R. \& Butler, B.M. (2002). The vacant quarter revisited: Late Mississippian abandonment of the Lower Ohio Valley. American Antiquity, 67(4), 625-641.

Costa, R.L., Jr. (1980). Age, sex, and antemortem loss of teeth in prehistoric Eskimo samples from Point Hope and Kodiak Island, Alaska. American Journal of Physical Anthropology, 53(4), 579587.

Crites, G.D. (1978). Paleoethnobotany of the Normandy Reservoir in the Upper Duck River Valley, Tennessee (Unpublished master's thesis). University of Tennessee, Knoxville.

Dowd, J.T. (2008). The Cumberland stone-box burials of middle Tennessee. Tennessee Archaeology, 3 (2), 163-180.

Dye, D.H. (2002, November). The 1936 University of Tennessee WPA-TVA excavations at the Link Farm Site (40HS6), Humphreys County, Tennessee. Paper presented at the annual meeting of the Southeastern Archaeological Conference, Biloxi.

Dye, D.H. (2003, January). The Link Farm Site (40HS6) in Regional Perspective. Paper presented at the annual meeting of Current Research in Tennessee Archaeology (CRITA), Nashville.

Dye, D.H. (2004, January). Buffer Zones, Warfare, and Settlement Patterning: Mississippian Polity Spacing in the Lower Tennessee Valley. Paper presented at the annual meeting of Current Research in Tennessee Archaeology (CRITA), Nashville.

Dye, D.H. (2007, January). The Duck River Cache and the Evolution of Mississippian Symbolic Weaponry. Paper presented at the annual meeting of Current Research in Tennessee Archaeology (CRITA), Nashville.

Dye, D.H. (2012, January). The Duck River Cache and Middle Mississippian Ritual Performance. Paper Presented at the annual meeting of Current Research in Tennessee Archaeology (CRITA), Nashville.

Dye, D.H. (2013). Trouble in the glen. In B.K. Means (Ed.), Shovel ready: Archaeology and Roose- velt's New Deal for America (pp. 129-146). Tuscaloosa: University of Alabama Press.

Dye, D.H. \& King, A. (2007. Desecrating the sacred mortuary shrines: Late prehistoric warfare in the Southeast. In R.J. Chacon, R.G. Mendoza (Eds.), North American indigenous warfare and ritual violence (pp. 160-181), Tucson: University of Arizona Press.

Duyar, I. \& Erdal, Y.S., (2003). A new approach for calibrating dental caries frequency of skeletal remains. Homo, 54(1), 57-70.

Emerson, T.E., Hedman, K.M., \& Simon, M.L. (2005). Marginal horticulturalists or maize agriculturalists? Archaeobotanical, paleopathological, and isotopic evidence relating to Langford Tradition maize consumption. Midcontinental Journal of Archaeology, 30(1), 67-118.

Emerson TE, KM Hedman, ML Simon, Matthew Fort 2020 Isotopic Confirmation of the Timing and Intensity of Maize Consumption in Greater Cahokia. American Antiquity, 85(2), 241-262.

Emerson, T.E., McElrath, D.L., \& Fortier, A.C. (2000). Late Woodland societies: Tradition and transformation across the Midcontinent. University of Nebraska Press, Lincoln.

Erdal, Y.S., \& Duyar, I (1999). A new correction procedure for calibrating dental caries frequency. American Journal of Physical Anthropology, 108 (2), 237-240.

Ferguson, R.B. (Ed.) (1972). The Middle Cumberland Culture. Vanderbilt University Publications in Anthropology, No 3, Nashville.

Ferraro, M., \& Vieira, A.R. (2010). Explaining gender differences in caries: a multifactorial approach to a multifactorial disease. International Journal of Dentistry, 2010 (Article ID 649643), 1-5. Fritz, G.J. (1990). Multiple pathways to farming in precontact eastern North America. Journal of World Prehistory, 4(4), 387-435.

Fritz, G.J. (1992). "Newer, better" maize and the Mississippian emergence: A critique of prime mover explanations. In W.I. Woods (Ed.), Late Prehistoric Agriculture: Observations from the Midwest, Studies in Illinois Archaeology 8, 19-43.

Fritz, G. J., and Lopinot, N. H. (2007). Native crops at early Cahokia: Comparing domestic and ceremonial contexts. Illinois Archaeology, 15-16, 90109.

Gremillion, K. J., Windingstad, J., Sherwood, S. (2008). Forest opening, habitat use, and food production on the Cumberland Plateau, Kentucky: Adaptive flexibility in a marginal setting. American Antiquity, 73(3), 387-411.

Grove, J.M. (2012). The Little Ice Age. New York: 
Routledge.

Hally, D.J. (1993). The territorial size of Mississippian chiefdoms. In J.B. Stoltman (Ed.), Archaeology of Eastern North America: Papers in Honor of Stephen Williams (pp. 143-168). Archaeological Report No. 25. Jackson, MS: Mississippi Department of Archives and History.

Han, K.X. \& Nakahashi ,T. (1996). A comparative study of ritual tooth ablation in ancient China and Japan. Anthropological Science, 104(1), 43-64.

Hart, J. P., \& Lovis, W.A. (2013). Reevaluating what we know about the histories of maize in northeastern North America: a review of current evidence. Journal of Archaeological Research, 21(2), 175-216.

Hix III, J.O., \& O'Leary, T.J.(1976). The relationship between cemental caries, oral hygiene status and fermentable carbohydrate intake. Journal of Periodontology, 47(7), 398-404.

Horton, W.A., Jacob, A.E., Green, W.R.M., Hillier, V.F., \& Drucker, D.B. (1985). The cariogenicity of sucrose, glucose and maize starch in gnotobiotic rats mono-infected with strains of the bacteria Streptococcus mutans, Streptococcus salivarius and Streptococcus milleri. Archives of Oral Biology, 30(11-12), 777-780.

Humphrey L., \& Bocaege E. (2008). Tooth avulsion in the Maghreb: chronological and geographical patterns. African Archaeological Review, 25(1-2), 109-23.

Hutchinson, D.L., Larsen, C.S., Schoeninger, M.J., \& Lynette Norr, L. (1998). Regional variation in the pattern of maize adoption and use in Florida and Georgia. American Antiquity, 63(3), 397-416.

Katz, S.H., Hediger, M.L., \& Valleroy, L.A. (1974).Traditional maize processing techniques in the New World. Science 184(4138), 765-773.

Kida, I.A., Åstrøm, A.N., Strand, G.V., \& Masalu, J.R. (2006). Clinical and socio-behavioral correlates of tooth loss: a study of older adults in Tanzania. BMC Oral Health, 6(1), 1-10.

King, A. (2002) Creek chiefdoms at the temporal edge of the Mississippian World. Southeastern Archaeology, 21(2), 221-226.

King, A., Dye, D.H., Muller, J., Scarry, J.F., Sullivan, L.P., \& Pauketat, T.R. (2007). Southeastern Ceremonial Complex: Chronology, Content, Contest. Tuscaloosa: The University of Alabama Press

King, A. \& Freer, J.A. (1995). The Mississippian Southeast: a world systems perspective. In M.S. Nassaney \& K.E. Sassaman (Eds.) Native American interactions: Multiscalar snalyses and interpretations in the Eastern Woodlands (pp. 266-288). Knoxville: University of Tennessee Press.
Kline, G., Crites, G.D., \& Faulkner, C.H. (1982). The McFarland Project: Early Middle Woodland settlement and subsistence in the Upper Duck River Valley in Tennessee. Miscellaneous Paper No. 8. Knoxville: Tennessee Anthropological Association.

Kuemin Drews, N. (2000). A bioarchaeological perspective of Mississippian period warfare in westcentral Tennessee (Unpublished master's thesis). Northern Illinois University, DeKalb, IL.

Krus, A.M. \& Cobb, C.R. (2018). The Mississippian fin de siècle in the middle Cumberland region of Tennessee. American Antiquity, 83(2), 302-319.

Krus, A.M. (2013). A chronology for warfare in the Mississippian Period (AD 1000-1500) (Unpublished doctoral dissertation). Indiana University, Bloomington.

Larsen, C.S. (1981). Skeletal and dental adaptations to the shift to agriculture on the Georgia coast. Current Anthropology, 22(4), 422-423.

Larsen, C.S. (1983). Behavioural implications of temporal change in cariogenesis. Journal of Archaeological Science, 10(1), 1-8.

Larsen, C.S., Shavit, R. \& Griffin, M.C. (1991). Dental caries evidence for dietary change: an archaeological context. In M.A. Kelly \& C.S. Larsen (Eds.) Advances in dental anthropology (pp. 179-202). Gainesville: University of Florida. Lewis, R.B., Stout, C., \& Wesson, C.B. (1998). The design of Mississippian towns. In R.B. Lewis and S. Stout (Eds.) Mississippian towns and sacred spaces: Searching for an architectural grammar (pp: 1-21). Tuscaloosa: University of Alabama Press.

Lingström, P., van Houte J., Kashket S. (2000). Food starches and dental caries. Critical Review of Oral Biological Medicine, 11(3), 366-380.

Lukacs, J.R. (1992). Dental paleopathology and agricultural intensification in South Asia: New evidence from Bronze Age Harappa. American Journal of Physical Anthropology, 87(2), 133-50.

Lukacs, J.R. (1995). The 'caries correction factor': a new method for calibrating dental caries rates to compensate for antemortem loss of teeth. International Journal of Osteoarchaeology, 5(2), 151-156.

Lukacs, J.R. ( 2007). Dental trauma and antemortem tooth loss in prehistoric Canary Islanders: prevalence and contributing factors. International Journal of Osteoarchaeology, 17(2), 157-173.

Lukacs, J.R. (2011). Sex differences in dental caries experience: clinical evidence, complex etiology. Clinical Oral Investigations, 15(5), 649-656.

Lukacs, J.R. and Largaespada, L.L., 2006. Explaining sex differences in dental caries prevalence: 
saliva, hormones, and "life-history" etiologies. American Journal of Human Biology, 18(4), 540555.

Lunn, A.R. (2013). WPA archaeology at the Slayden site, Humphreys County, Tennessee. In J.L. Cordell, J.F. Doershuk, D.H. Dye, S.W. Hammerstedt, J.R. Johnson, K. Kiernan, \& G.D. Lattanzi (Eds.) Shovel ready: Archaeology and Roosevelt's new deal for America (pp. 147-164). Tuscaloosa: University of Alabama Press.

McMahan, J.D. (1983). Paleoethnobotany of the Late Woodland Mason Phase in the Elk and Duck River Valleys, Tennessee (Unpublished master's thesis). University of Tennessee, Knoxville.

Mainfort Jr., R.C. (1996). Late period chronology in the Central Mississippi Valley: A Western Tennessee perspective. Southeastern Archaeology, 15 (2), 172-181.

Mainfort, Jr., R.C. \& Moore, M.C. (1998). Graves Lake: A Late Mississippian Period village in Lauderdale County, Tennessee. In M.J. O'Brien \& R.C. Dunnell (Eds.) Changing perspectives on the archaeology of the Central Mississippi Valley (pp. 99-123). Tuscaloosa: University of Alabama Press.

Mann, M.E., Zhang, Z., Rutherford, S., Bradley, R.S., Hughes, M.K., Shindell, D., Ammann, C., Faluvegi, G., \& Ni, F. (2009). Global signatures and dynamical origins of the Little Ice Age and Medieval Climate Anomaly. Science, 326(5957), 1256-1260.

Márquez-Grant, N. (2009). Caries correction factors applied to a Punic (6th-2nd BC) population from Ibiza (Spain). Bulletin of the International Association for Paleodontology, 3(1), 20-29.

Meeks, S.C., \& Anderson, D.G. (2013). Drought, subsistence stress, and population dynamics: Assessing Mississippian abandonment of the vacant quarter. In J.D. Wingard, \& S.E. Hayes (Eds.) Soils, climate, and society: Archaeological investigations in ancient America. (pp 61-83). Boulder: University Press of Colorado.

Moore, M.C. \& Smith, K.E. (2001). Archaeological Excavations at the Rutherford-Kizer Site: A Mississippian Mound Centerin Sumner County, Tennessee. Tennessee Department of Environment and Conservation, Division of Archaeology, Research Series No.13, Nashville.

Müller, A. \& Hussein, K. (2017). Meta-analysis of teeth from European populations before and after the 18th century reveals a shift towards increased prevalence of caries and tooth loss. Archives of Oral Biology, 73(1), 7-15.
Naftz, D.L., Klusman, R.W., Michel, R.L., Schuster, P.F., Reddy, M.M., Taylor, H.E., Yanosky, T.M. \& McConnaughey, E.A. (1996). Little Ice Age evidence from a south-central North American ice core, USA. Arctic and Alpine Research, 28(1), 35-41.

Nash, C.H. (1968). Residence mounds: An Intermediate Middle Mississippian settlement pattern. Memphis State Anthropological Research Center Occasional Papers, No. 2, Memphis.

Nassaney, M S. (2001). The historical-processual development of Late Woodland societies. In T.R. Pauketat (Ed.) The archaeology of traditions: Agency and history before and after Columbus (pp. 157-173). Gainesville: University Press of Florida.

Nelson, G.C., Lukacs, J.R. \& Yule, P. (1999). Dates, caries, and early tooth loss during the Iron Age of Oman. American Journal of Physical Anthropology, 108(3), 333-343.

Newbrun, E. (1979). Dietary carbohydrates: their role in cariogenicity. Medical Clinics of North America, 63(5), 1069-1086.

Niessen, L.C., \& Weyant, R.J. (1989). Causes of tooth loss in a veteran population. Journal of Public Health Dentistry, 49(1), 19-23.

Ong, G. (1998). Periodontal disease and tooth loss. International Dental Journal, 48(S3), 233-238.

O'Sullivan, DM, \& Tinanoff N. (1993). Maxillary anterior caries associated with increased caries risk in other primary teeth. Journal of Dental Research, 72(12), 1577-80.

Peregrine, P.N. (2013). Archaeology of the Mississippian culture: A Research Guide. New York: Routledge.

Peres, T.M. (2017). Foodways archaeology: a decade of research from the southeastern United States. Journal of Archaeological Research, 25(4), 421-460.

Pollard, M.A. (1995). Potential cariogenicity of starches and fruits as assessed by the plaquesampling method and an intraoral cariogenicity. Caries Research, 29(1), 68-74.

Pollard, M. A., Cleaton-Jones, P., Smit, A., \& Sam, C. (1997). The plaque acidogenic response to carbohydrates of children from three ethnic groups in South Africa. International Dental Journal, 47(1), 39-44.

Powell, M.L. (1985). The analysis of dental wear and caries for dietary reconstruction. In R.I. Gilbert, \& J.H. Mielke (Eds.), The analysis of prehistoric diets (pp. 307-338). New York: Academic Press. 
Raviele, M.E. (2011). Experimental assessment of maize phytolith and starch taphonomy in carbonized cooking residues. Journal of Archaeological Science, 38(10), 2708-2713.

Russell, S.L., Gordon, S., Lukacs, J.R. \& Kaste, L.M. (2013). Sex/Gender differences in tooth loss and edentulism: historical perspectives, biological factors, and sociologic reasons. Dental Clinics, 57 (2), 317-337.

Scarry, C.M. (1993). Variability in Mississippian crop production strategies. In C.M. Scarry (Ed.), Foraging and farming in the Eastern Woodlands (pp. 78-90). Gainesville: University Press of Florida.

Schroedl, G.F. (1998). Mississippian towns in the eastern Tennessee Valley. In R B. Lewis \& C. B Stout (Eds.) Mississippian towns and sacred spaces: Searching for an architectural grammar (pp. 64-92). Tuscaloosa: University of Alabama Press.

Scopa Kelso, R. (2018). Mississippian subadults from the Middle Cumberland and Eastern regions of Tennessee: Biological indicators of population interaction. American Journal of Physical Anthropology, 166(2), 417-432.

Shaffer, J.R., Polk, D.E., Feingold, E., Wang, X., Cuenco, K.T., Weeks, D.E., Rebecca S. DeSensi, R.S., Weyant, R.J., Crout, R., \& McNeil, D.W. (2013). Demographic, socioeconomic, and behavioral factors affecting patterns of tooth decay in the permanent dentition: principal components and factor analyses. Community Dentistry and Oral Epidemiology, 41(4), 364-373.

Shea, A.B. (1977). Comparison of Middle Woodland and Early Mississippian subsistence patterns: analysis of plant remains from an archaeological site in the Duck River Valley, Tennessee, supplemented by the potentially exploitable native flora (Unpublished doctoral dissertation). University of Tennessee, Knoxville.

Smith, K.E. (1992). The Middle Cumberland Region: Mississippian archaeology in North Central Tennessee (Unpublished doctoral dissertation). Vanderbilt University, Nashville, Tennessee.

Smith, K.E. (2010, October). Oh where, oh where, did everyone go?: The 15th century population dispersal from Middle Tennessee. Paper presented at the annual Midwestern Archaeological Conference, Bloomington, Indiana.

Smith, M.O. \& Betsinger, T.K. (2019). Dental caries as an archaeological problem-solving tool: Reconstructing subsistence patterns in Late Prehistoric west-central Tennessee. Dental Anthropolo$g y, 32(2), 51-66$.

Somerville, A.D., Goldstein, P.S., Baitzel,S.I., Bru- welheide, K.L., Dahlstedt, A.C., Yzurdiaga,L. Raubenheimer, S., Knudson, K.J., \& Schoeninger, M.J. (2015). Diet and gender in the Tiwanaku colonies: stable isotope analysis of human bone collagen and apatite from Moquegua, Peru. American Journal of Physical Anthropology, 158 (3), 408-422.

Stahle, D.W., \& Cleaveland, M.K. (1994). TreeRing reconstructed rainfall over the Southeastern U.S.A. During the Medieval Warm Period and Little Ice Age. In M.K. Hughes and H.F. Diaz (Eds.). The Medieval Warm Period (pp. 199-212). Dordrecht: Springer.

Turner, C.G. (1979). Dental anthropological indications of agriculture among the Jomon people of central Japan. X. Peopling of the Pacific. American Journal of Physical Anthropology, 51(4), 619635.

van der Velden, U., Amaliya, A., Loos, B.G., Timmerman, M.F., van der Weijden, F.A., Winkel, E.G., \& Abbas, A. (2015). Java project on periodontal diseases: causes of tooth loss in a cohort of untreated individuals. Journal of Clinical Periodontology, 42(9), 824-831.

VanDerwarker, A.M., Bardolph, D.N., \& Scarry, C.M. (2017). Maize and Mississippian beginnings. In G.D. Wilson (Ed.), Mississippian beginnings (pp. 29-70). Gainesville: University Press of Florida.

Walter, B.S., DeWitte, S.N. \& Redfern, R.C. (2016). Sex differentials in caries frequencies in Medieval London. Archives of Oral Biology, 63, 32-39.

Wamsley, B.M. (2018). Mortuary patterns in westcentral Tennessee: Contextualizing historic field data from nine Mississippian period sites (Unpublished master's thesis). Illinois State University, Normal.

Watson, J.T., 2008. Prehistoric dental disease and the dietary shift from cactus to cultigens in northwest Mexico. International Journal of Osteoarchaeology, 18(2), 202-212.

Williams, S. (1983). Some ruminations on the current strategy of research in the Southeast. Southeastern Archaeological Conference (Bulletin 21), 7281.

Williams, S. (1990). The vacant quarter and other late events in the Lower Valley. In D.H. Dye, \& C.A. Cox, (Eds.), Towns and temples along the Mississippi (pp. 170-180). Tuscaloosa: University of Alabama Press.

Wilson, G. D. (2017) Mississippian beginnings. Gainesville: University Press of Florida.

Wilson, G.D. \& L.P. Sullivan. (2017). Mississippian origins: from emergence to beginnings. In G.D. 
Wilson (Ed.) Mississippian beginnings (pp. 1-28). Gainesville: University Press of Florida.

Wilson, J.J. (2017, April). The development of the mid-continental U.S. vacant Quarter: the impact of aggression, warfare, and climate change on late pre-Columbian population dynamics. Paper presented at the annual meetings of the American Association of Physical Anthropologists, New Orleans, La.

Worne, H. (2011). Conflicting spaces: biological and geophysical perspectives on warfare in the Middle Cumberland region of Tennessee (Unpublished doctoral dissertation). Binghamton University, New York. 